\title{
Téoros
}

Revue de recherche en tourisme

\section{Dites-moi votre événement et je vous dirai votre clientèle}

\section{Pierre Bellerose}

Volume 5, numéro 2, juillet 1986

Tourisme, fêtes et événements

URI : https://id.erudit.org/iderudit/1080571ar

DOI : https://doi.org/10.7202/1080571ar

Aller au sommaire du numéro

Éditeur(s)

Université du Québec à Montréal

ISSN

0712-8657 (imprimé)

1923-2705 (numérique)

Découvrir la revue

Citer cet article

Bellerose, P. (1986). Dites-moi votre événement et je vous dirai votre clientèle. Téoros, 5(2), 9-10. https://doi.org/10.7202/1080571ar d'utilisation que vous pouvez consulter en ligne.

https://apropos.erudit.org/fr/usagers/politique-dutilisation/ 


\section{Dites-moi votre événement et je vous dirai votre clientèle \\ par Pierre Bellerose *}

Depuis l'Expo 67 surtout, les événements a caractère touristique ont toujours pris une grande importance au Québec et plus précisément à Montréal. On peut penser, entre autres, aux Olympiques de 76, aux Floralies internationales, à Ramses II et aux nombreux festivals internationaux de nature récurente ou ponctuelle.

Cependant, jusqu'à tout récemment, aucune statistique sérieuse ${ }^{(1)}$ n'est venue préciser le type de clientéle de ce genre d'événement, ni ponctuer l'attrait que peuvent procurer ces événements sur la clientèle touristique.

Pour pallier à cette faiblesse d'autant plus importante que Montréal met de plus en plus l'emphase sur les evvénements internationaux dans sa stratégie touristique, le ministère fédéral des Communications et le ministère de l'Expansion industrielle fégionale (Tourisme Canada) décidèrent de mandater la firme Econosult pour effectuer une collecte de données aupres de la clientèle de neuf (9) évênements d'envergure de la région de Montréal. Ce sont: le Festival international de Jazz de Montréal, le Festival Juste pour Rire, le Festival de Théátre des Amériques, le Festival de Théătre Jeunes Publics, l'Exposition Ramsès II, le Grand Prix Automobile de Montréal,
l'Exposition Picasso, le Tournoi de Tennis Players et le Festival des Films du Monde ${ }^{2}$,

Cette vaste étude ${ }^{37}$ de plus de 5,000 entrévues pour la région de Montréal permet de dresser un portrait représentatif des différents types de clientèles. C'est le premier pas vers une meilleure compréhension des interrelations entre ces attraits particuliers et le monde touristique. Cela deviendra aussi un outil essentiel pour les administrateurs de ces événements, permettant enfin d'avoir des previsions sur la matière première.

\section{Origine de la clientèle}

Si l'on examine attentivement l'origine de la clientèle de ces neuf (9) événements à l'aide du tableau 1, on constate des écarts très grands d'un à l'autre. Ainsi, il appert que le Grand Prix Automobile de Montréal suivi de l'Exposition Picasso attirèrent les clientèles hors-Québec les plus imposantes avec respectivement $45.1 \%$ et $34.5 \%$ du total. A l'autre extrème, le Festival Juste pour Rire fut celui qui en accueillit le moins avec seulement $4.8 \%$. D'une façon générale, on peut affirmer que les événements sportifs (Grand Prix, Tennis) et ponctuels (Ramsès II, Picasso) ont reçu une clientèle hors-Québec beaucoup plus importante que les événements culturels à caractère récurent (la seule exception est le Théâtre des Amériques mais comme c'est l'échantillon le plus faible, on peut l'éliminer de notre analyse). Cette statistique surprenante permet de relativer maintenant la place des grands festivals internationaux dans l'échiquier touristique. Évidemment, l'interprétation de ces résultats doit aussi tenir compte de plusieurs facteurs dont les efforts de promotion effectués pour chacun des événements... ce que pour l'instant nous ne sommes pas en mesure de faire.

\section{But du séjour}

Le tableau 2 nous permet de pousser plus loin la réflexion en indiquant le potentiel attractif des événements. En effet, on peut isoler les répondants venant de l'extérieur de Montréal qui sont venus expressément pour l'événement. Cette statistique, au point de vue touristique, est essentielle puisqu'elle permet de voir si un événement attire vraiment une clientèle additionnelle qui ne viendrait pas de ce dit événement. Ainsi, on apprend que $90 \%$ de la clientèle provenant du Grand Prix Automobile de Montréal s'est retrouvée à Montréal en raison de l'événement alors que le pourcentage le plus bas se retrouve pour les événements ponctuels (Picasso et Ramsès II) avec respectivement $37.5 \%$ et $32.1 \%$.

Tableau 1

Origine de la clientèle

\begin{tabular}{|c|c|c|c|c|c|c|c|c|c|}
\hline Type de clientèle & $\begin{array}{l}\text { Théatre } \\
\text { Ameriques }\end{array}$ & $\begin{array}{l}\text { Théâtre } \\
\text { Jeunes } \\
\text { Publics }\end{array}$ & $\begin{array}{l}\text { Festival } \\
\text { Jazz }\end{array}$ & $\begin{array}{c}\text { Festival } \\
\text { Juste } \\
\text { pour Rire }\end{array}$ & $\begin{array}{c}\text { Exposition } \\
\text { Ramses } \\
\text { II }\end{array}$ & $\begin{array}{l}\text { Exposition } \\
\text { Picasso }\end{array}$ & $\begin{array}{c}\text { Grand } \\
\text { Prix } \\
\text { Automobile }\end{array}$ & $\begin{array}{l}\text { Tennis } \\
\text { Player's }\end{array}$ & $\begin{array}{c}\text { Festival } \\
\text { Films du } \\
\text { Monde }\end{array}$ \\
\hline Région de Montráal \% & 78.9 & 82.2 & 81.1 & 91.2 & 61.5 & 52 & 44.8 & 77.9 & 85.6 \\
\hline $\begin{array}{l}\text { Province de Québec \% } \\
\text { (excepté Mtl) }\end{array}$ & 7.2 & 11.7 & 9.2 & 4.0 & 19.1 & 13.5 & 10.1 & 10.1 & 5.6 \\
\hline $\begin{array}{l}\text { Canada } \\
\text { (excepté Québec) }\end{array}$ & 7.6 & 3.8 & 4.6 & 2.6 & 9.7 & 15.6 & 22.1 & 7.7 & 4.2 \\
\hline Etats-Unis & 1.3 & 1.5 & 3.0 & 1.4 & 7.3 & 14.5 & 20.8 & 2.8 & 2.6 \\
\hline Autres & 5.0 & 1,8 & 2.1 & 0.8 & 2.4 & 4.4 & 2.2 & 1.5 & 2.0 \\
\hline $\begin{array}{l}\text { Sous-total } \\
\text { hors-Québec }\end{array}$ & $13.9 \%$ & $7.1 \%$ & $9.7 \%$ & $4.8 \%$ & $19.4 \%$ & $34.5 \%$ & $45.1 \%$ & $12.0 \%$ & $8.8 \%$ \\
\hline $\begin{array}{l}\text { Nombre } \\
\text { (echantillon) }\end{array}$ & 237 & 266 & 1165 & 499 & 660 & 743 & 495 & 575 & 577 \\
\hline
\end{tabular}

"Pierne Bellerose est professeur en fourisme au Cegep

de Gramby 


\begin{tabular}{|c|c|c|c|c|c|c|c|c|c|c|}
\hline But du voyage & & $\begin{array}{l}\text { Théatre } \\
\text { Amériques }\end{array}$ & $\begin{array}{l}\text { Thedatre } \\
\text { Jeunes } \\
\text { Publics }\end{array}$ & $\begin{array}{c}\text { Festival } \\
\text { Jazz }\end{array}$ & $\begin{array}{c}\text { Festival } \\
\text { Juste } \\
\text { pour Rire }\end{array}$ & $\begin{array}{c}\text { Exposition } \\
\text { Ramsès } \\
\text { II }\end{array}$ & $\begin{array}{c}\text { Exposition } \\
\text { Picasso }\end{array}$ & $\begin{array}{c}\text { Grand } \\
\text { Prix } \\
\text { Automobile }\end{array}$ & $\begin{array}{l}\text { Tennis } \\
\text { Player's }\end{array}$ & $\begin{array}{c}\text { Festival } \\
\text { Films du } \\
\text { Monde }\end{array}$ \\
\hline \% congres/travail & $\begin{array}{l}\mathrm{T} \\
\mathrm{O}: \\
\mathrm{C}: \\
\mathrm{A}: \\
\mathrm{E}:\end{array}$ & $\begin{array}{r}42,9 \\
23,6 \\
66.7 \\
-\overline{7}\end{array}$ & $\begin{array}{r}8,0 \\
10,3 \\
0,0 \\
25,0 \\
0,0\end{array}$ & $\begin{array}{r}15,5 \\
11,0 \\
9,5 \\
31,1 \\
23,3\end{array}$ & $\begin{array}{r}7,4 \\
-5,0 \\
25,0\end{array}$ & $\begin{array}{r}6,5 \\
5,1 \\
8,6 \\
6,5 \\
31,8\end{array}$ & $\begin{array}{r}12,6 \\
4,4 \\
11,1 \\
18,7 \\
21,9\end{array}$ & $\begin{array}{r}5,6 \\
- \\
5,3 \\
5,6 \\
25,0\end{array}$ & $\begin{array}{r}2,6 \\
1,5 \\
- \\
4,8 \\
20,0\end{array}$ & $\begin{array}{r}13,3 \\
6,3 \\
24,0 \\
- \\
25,0\end{array}$ \\
\hline \% evênement & $\begin{array}{l}\text { T: } \\
\mathrm{Q}: \\
\mathrm{C}: \\
\mathrm{A}: \\
\mathrm{E}:\end{array}$ & $\begin{array}{l}53,1 \\
70,6 \\
33,3 \\
66,7 \\
58,3\end{array}$ & $\begin{array}{l}66,0 \\
69,0 \\
90,0 \\
25,0 \\
60,0\end{array}$ & $\begin{array}{l}61,9 \\
74,8 \\
68,3 \\
46,7 \\
23,3\end{array}$ & $\begin{array}{c}44,1 \\
61,8 \\
30,0 \\
30,0 \\
-\end{array}$ & $\begin{array}{r}37,5 \\
56,1 \\
26,8 \\
16,4 \\
-\end{array}$ & $\begin{array}{r}32,1 \\
57,0 \\
31,9 \\
18,7 \\
2,4\end{array}$ & $\begin{array}{l}90,2 \\
94,0 \\
93,0 \\
90,7 \\
41,7\end{array}$ & $\begin{array}{c}72,2 \\
80,6 \\
81,1 \\
57,1 \\
-\end{array}$ & $\begin{array}{l}53,0 \\
56,2 \\
60,0 \\
53,3 \\
33,3\end{array}$ \\
\hline
\end{tabular}

Legende:

$T=$ ensemble des répondants venant de l'extérieur de Montréal

$\mathrm{Q}=$ répondants québécois lexcluant Montréall
$\mathrm{C}$ - répondants canadiens lexcluant Québecl

$\mathrm{A}=$ repondants americains

$E=$ repondants étranqers

Tableau 3

Dépenses de la clientèle lors du séjour à Montréal

\begin{tabular}{|c|c|c|c|c|c|c|c|c|c|c|}
\hline Variables & & $\begin{array}{l}\text { Théatre } \\
\text { Amériques }\end{array}$ & $\begin{array}{l}\text { Théatre } \\
\text { Jeunes } \\
\text { Publics }\end{array}$ & $\begin{array}{l}\text { Festival } \\
\text { Jazz }\end{array}$ & $\begin{array}{c}\text { Festival } \\
\text { Juste } \\
\text { pour Rire }\end{array}$ & $\begin{array}{c}\text { Exposition } \\
\text { Ramses } \\
\text { II }\end{array}$ & $\begin{array}{l}\text { Exposition } \\
\text { Picasso }\end{array}$ & $\begin{array}{c}\text { Grand } \\
\text { Prix } \\
\text { Automobile }\end{array}$ & $\begin{array}{l}\text { Tennis } \\
\text { Player's }\end{array}$ & $\begin{array}{c}\text { Festival } \\
\text { Films du } \\
\text { Monde }\end{array}$ \\
\hline $\begin{array}{l}\text { Dépenses totales: } \\
\text { moyennes } \$\end{array}$ & $\begin{array}{c}\text { T: } \\
\text { M: } \\
\text { Q: } \\
\text { C: } \\
\text { A: } \\
\text { E: }\end{array}$ & $\begin{array}{r}610.77 \\
19.38 \\
282.35 \\
484.06 \\
483.33 \\
1.263 .75\end{array}$ & $\begin{array}{c}306.69 \\
14.94^{*} \\
134.32^{*} \\
267.00 \\
363.33^{*} \\
764.60\end{array}$ & $\begin{array}{c}529.41^{*} \\
30.51 \\
300.53 \\
514.85 \\
790.82 \\
1,055.82^{*}\end{array}$ & $\begin{array}{c}471.74^{\circ} \\
30.22 \\
242.94 \\
724.85 \\
790.00^{\circ} \\
793.00\end{array}$ & $\begin{array}{c}489.51^{*} \\
26.77 \\
242.08^{*} \\
500.91 \\
857.98 \\
1,853.24\end{array}$ & $\begin{array}{r}607.17 \\
24.69 \\
204.93 \\
522.70 \\
731.03 \\
1,630.61\end{array}$ & $\begin{array}{c}553.56^{*} \\
56.50 \\
240.90 \\
429.88^{*} \\
689.08 \\
1.703 .64^{*}\end{array}$ & $\begin{array}{c}545.03 \\
29.14 \\
242.66^{*} \\
355.23^{*} \\
915.57^{\circ} \\
1.617 .33\end{array}$ & $\begin{array}{c}677.44^{\circ} \\
19.90 \\
377.03 \\
574.16^{\circ} \\
944.73 \\
1.377 .90^{\circ}\end{array}$ \\
\hline $\begin{array}{l}\text { Nombre moyen } \\
\text { de personnes } \\
\text { incluses dans } \\
\text { ces dépenses }\end{array}$ & $\begin{array}{l}\mathrm{T}: \\
\mathrm{M}: \\
\mathrm{Q}: \\
\mathrm{C}: \\
\mathrm{A}: \\
\mathrm{E}:\end{array}$ & $\begin{array}{l}1,2 \\
1,2 \\
1,3 \\
1,2 \\
1,3 \\
1,0\end{array}$ & $\begin{array}{l}1,9 \\
2,5 \\
2,6 \\
2,0 \\
1,3 \\
1,8\end{array}$ & $\begin{array}{l}1,3 \\
1,3 \\
1,4 \\
1,3 \\
1,4 \\
1,0\end{array}$ & $\begin{array}{l}1.5 \\
1,5 \\
1,6 \\
1,4 \\
1.9 \\
1,0\end{array}$ & $\begin{array}{l}1,8 \\
1,8 \\
1,8 \\
1,8 \\
3,8 \\
1,4\end{array}$ & $\begin{array}{l}1,6 \\
1,5 \\
1,8 \\
1,5 \\
1,8 \\
1,2\end{array}$ & $\begin{array}{l}1,6 \\
1,3 \\
1,6 \\
1,5 \\
1,7 \\
1,1\end{array}$ & $\begin{array}{l}1,8 \\
1,5 \\
1,6 \\
1,9 \\
2,1 \\
1,3\end{array}$ & $\begin{array}{l}1,5 \\
1,2 \\
1,3 \\
1,3 \\
1,4 \\
1,8\end{array}$ \\
\hline $\begin{array}{l}\text { Depenses totales } \\
\text { per capita \$ }\end{array}$ & $\begin{array}{l}\mathrm{T}: \\
\mathrm{M}: \\
\mathrm{Q}: \\
\mathrm{C}: \\
\mathrm{A}: \\
\mathrm{E}:\end{array}$ & $\begin{array}{r}508.33 \\
16.15 \\
217.19 \\
403.38 \\
371.79 \\
1.263 .75\end{array}$ & $\begin{array}{c}161.41 \\
5.97 \\
51.66 \\
133.50 \\
279.48 \\
424.78\end{array}$ & $\begin{array}{c}407.23^{\circ} \\
20.47 \\
214.66 \\
396.11 \\
564.87 \\
1,055.82^{*}\end{array}$ & $\begin{array}{c}314.49^{*} \\
20.14 \\
151.84 \\
519.89^{\circ} \\
415.79^{\circ} \\
793.00^{\circ}\end{array}$ & $\begin{array}{c}271.95^{*} \\
14.87 \\
113.37^{*} \\
278.28 \\
225.78 \\
1.323 .74\end{array}$ & $\begin{array}{r}379.37 \\
16.46 \\
113.85 \\
348.46 \\
406.13 \\
1,358.84\end{array}$ & $\begin{array}{c}345.97^{\circ} \\
43.46 \\
150.56 \\
285.59^{*} \\
405.34 \\
1,548.76\end{array}$ & $\begin{array}{c}302.79 \\
19.43 \\
151.66^{\circ} \\
186.95^{*} \\
435.98^{\circ} \\
1.244 .10\end{array}$ & $\begin{array}{c}451.62^{\circ} \\
16.58^{*} \\
290.02 \\
441.66^{*} \\
674.81 \\
765.50^{\circ}\end{array}$ \\
\hline
\end{tabular}

* Cas extrémes omis

Légende:

$T=$ ensemble des répondants venant de l'extérieur de Montréal

$\mathrm{M}=$ répondants montréalais (dépenses pour 1 journée)

$\mathrm{Q}=$ répondants québécois (excluant Montréal)
$\mathrm{C}=$ répondants canadiens (excluant Québecl
$\mathrm{A}=$ répondants américains
$\mathrm{E}=$ répondants étrangers
On remarque que ce sont les deux événements sportifs (Grand Prix et Tennis) qui ont obtenu la part la plus large de répondants $(90.2 \%$ et $72.2 \%)$ ayant affirmé être venus à Montréal en premier lieu pour l'événement. Il faut cependant ajouter que les expositions Picasso et Ramsès II ont attiré une clientèle beaucoup plus nombreuse que les événements sportifs. Même si pour les deux expositions, le but du voyage, pour la majorité (soit environ les 2/3). n'est pas l'événement, ils ont sans doute eu l'impact touristique le plus grand.

\section{Les dépenses}

Enfin, le tableau 3 permet de visualiser les dépenses encourues par les répondants lors des deux séjours à Montréal. Les dépenses totales per capita (en \$) vont de $161,41 \$$ (Festival de Theátre Jeunes Publics qui a une clientèle essentiellement montréalaise) à 412,625 pour le Festival des Films du Monde. Dans une analyse plus poussée des dépenses, on devra tenir compte évidemment du but du voyage (voir tableau 2) et du nombre de personnes attirées vers les différents événements (chiffres que nous né possédons pas).

Il semble évident, à premiere vue, que les événements sportifs ont un impact touristique beaucoup plus important que l'on pensait et qu'il faudrait en tenir compte maintenani dans notre stratégie. Cependant, ill n'en demeure pas moins que les ếvénements culturels sont devenus la carte maitresse en matière touristique pour Montréal et permettent, au fur et à mesure, de donner à cette ville un visage unique. Toutefois, on note que les festivals culturels attirent, en trés grande majorité (toujours plus de $80 \%$ ) une clientèle provenant de
Montréal et n 'ont sans doute pas pu aller chercher tout le potentiel possible au niveau touristique. Il faudrait peut-être chercher des raisons au niveau des efforts de promotion qui sont très differents d'un organisme à l'autre et souvent d'une année à l'autre. $f$

Notes

111 Une des sevles ftudes existantes ast le rapport de recherche de François Colbert et Jacques M. Boisvert, Etude de certaines dimensions economi ques des activités a caractère euturel, mai 1985 On traite, dans ce document, d'une facon tres inte. ressante, entre autres du cas du Fostival de Jazz de Montreal

(2) II esi a noter que cette btude est une constituante d'une enquéte encore plus waste traitant aussi des villes de Guelph et de Charlottetown. Pour plus de détsils, voir: The Tourism and Culture Pilot Project: An Integrated Analysis, Onawa, decembre 1985.

(3) ECONOSULT, Resultats d"un sondage aupress de La clienteble de neuf événements de rendez-vous Montréal 1985, novembre 1995. 